\title{
Psychische Belastung in der Tierarztpraxis
}

\author{
Karolina Kantor
}

\begin{abstract}
Die große Verantwortung und die vielfältigen Aufgaben in der tierärztlichen Praxis führen immer wieder zu psychischen Belastungen. Aber wovon hängt es ab, ob man die psychisch belastenden Situationen besser oder schlechter bewältigen kann und wie geht man am besten mit ihnen um, um ernsthafte Folgen für die seelische Gesundheit zu vermeiden?
\end{abstract}

Neben dem berufsbedingten Wunsch zu helfen und zu heilen, spüren viele Tierärzte heutzutage auch einen wirtschaftlichen Druck. Dieser entsteht, weil die Behandlung in der Regel vom Tierhalter selbst bezahlt wird, was nicht selten zu überhöhten oder gar unrealistischen Ansprüchen an den Tierarzt führt. Für diese Ansprüche gibt es unterschiedliche Gründe: Manchmal liegt es an den Therapiekosten, manchmal auch an mangelnden medizinischen Kenntnissen der Tierhalter oder an der Vorstellung, dass die Tiermedizin weniger kompliziert ist als die Humanmedizin. Eine zusätzliche Belastung kann entstehen, weil in der Tierarztpraxis nicht nur das Leid der Patienten, sondern auch das der Tierhalter direkt miterlebt wird.

In einer klinischen Studie mit über 11000 US-amerikanischen Tierärzten konnte nachgewiesen werde, dass bei 1 von 11 Tierärzten psychische Beschwerden auftreten und 1 von 6 sogar an Suizidgedanken leiden [1]. Dies zeigt, dass es sich um ein ernst zu nehmendes Thema handelt. Doch wovon hängt es ab, ob man die psychische Belastung besser oder schlechter bewältigen kann?

\section{Psychische Vulnerabilität - Was macht uns verletzlich?}

Jeder Mensch hat eine gewisse Vulnerabilität, d.h. die Prädisposition, psychische oder emotionale Beschwerden zu entwickeln. Diese ist u.a. genetisch bedingt. Je nachdem, wie hoch unser Vulnerabilitätslevel ist, desto weniger Stress brauchen wir, um die „kritische Grenze“ zu erreichen. So kann man vereinfacht das „VulnerabilitätsStress-Modell“ erklären, das oft grafisch als unterschiedlich gefüllte Fässer dargestellt wird. Aber welche Faktoren haben noch Einfluss auf unsere Stressresistenz?

\section{Empathiefähigkeit als Freund und Feind}

Bestimmte Persönlichkeitseigenschaften können zu einer erhöhten Vulnerabilität führen. Menschen, die sich für die Tiermedizin entscheiden, zeigen häufig eine be- sonders ausgeprägte Empathiefähigkeit. Diese kann der beste Freund und der schlimmste Feind eines Mediziners sein. Sie hilft enorm, eine gute Beziehung zu Patienten und Angehörigen aufzubauen und führt meistens dazu, dass man als Arzt sehr beliebt ist. Die dunkle Seite der Empathie ist jedoch, dass uns das Schicksal unserer Patienten psychisch belastet und wir nach dem Feierabend nicht „einfach abschalten“ können. Auch Perfektionismus ist eine in tierärztlichen Kreisen verbreitete Eigenschaft, die sehr hilfreich sein kann, oft jedoch leider mit der Konsequenz, dass man sich selbst enorm unter Druck setzt. Misserfolge werden von perfektionistischen Menschen viel intensiver erlebt.

Hinzu kommen soziale Aspekte wie Familie, Freundeskreis und soziale Situation, aber auch die „Work-LifeBalance“. Wenn es gleichzeitig in einem von diesen Bereichen und im Berufsleben Belastungsfaktoren gibt, kann es zu einer schnelleren psychischen Erschöpfung führen.

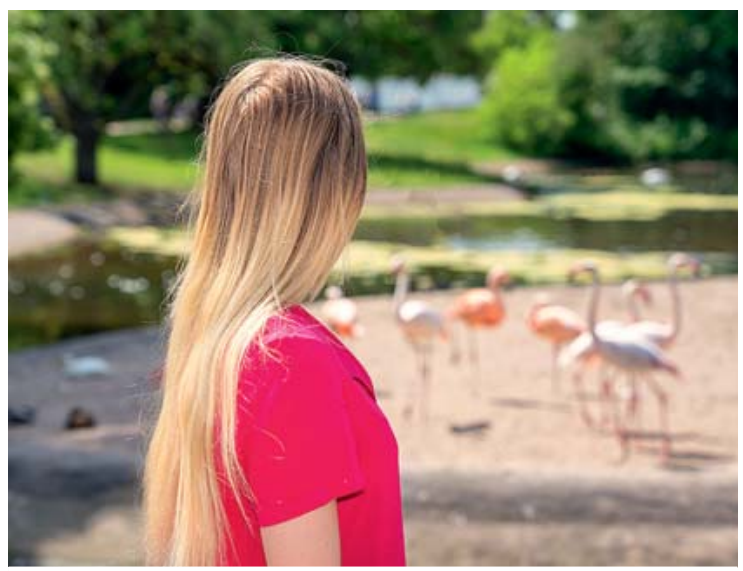

Abb. 1 Ob jemand gut oder schlecht mit Belastungen umgehen kann, hängt auch vom Vulnerabilitätslevel ab. Bestimmte Persönlichkeitseigenschaften und soziale Aspekte wie Familie, Freunde und Work-Life-Balance haben hier Einfluss. Quelle: Kirsten Oborny, Thieme Gruppe 


\section{Blinde Flecken}

In der Psychotherapie spricht man von „blinden Flecken“. Es sind die Eigenschaften, Erfahrungen und Gedanken, die uns unbewusst stark geprägt haben. Oft entdeckt man diese erst im Berufsleben, wenn man mit einer Situation konfrontiert wird, die in uns eine intensive Reaktion auslöst. Es ist deswegen wichtig, eigene „blinde Flecken“ zu identifizieren, denn nur so können Sie versuchen, sie entweder in den Berufsalltag zu integrieren oder, wenn sie sehr belastend sind und die normale Berufsausübung erschweren, therapeutisch zu bearbeiten.

Im Folgenden möchte ich einige Beispiele für psychisch belastende Situationen im Berufsleben des Tierarztes näher beleuchten, die mir aus psychiatrischer Sicht wichtig erscheinen.

\section{Wenn man an die eigenen Grenzen stößt...}

Immer wieder wird man im tierärztlichen Beruf mit Situationen konfrontiert, die einen psychisch besonders belasten. Wenn Sie den Eindruck haben, dass ein bestimmter Fall für sie psychisch schwer zu ertragen ist, versuchen Sie zunächst zu identifizieren, woran es liegen könnte. Je nachdem, zu welchem Ergebnis Sie kommen, ist es abzuwägen, ob Sie (ggf. mithilfe einer Supervision) den Patienten trotzdem weiterbehandeln möchten. Als Tierarzt ist man nicht automatisch verpflichtet, um jeden Preis über seine Grenzen zu gehen. Wenn Sie sich nicht in der Lage fühlen, den Patienten zu behandeln oder mit dem Tierhalter umzugehen, übergeben Sie ihn an einen Kollegen oder sprechen Sie es bei Ihrem Vorgesetzten an.

Wenn Sie erst während oder nach Abschluss der Behandlung feststellen, dass der Fall für Sie übermäßig belastend war, bieten sich verschiedene Ansätze an. Zum einen können Sie versuchen, selbst die Ursache Ihrer Reaktion zu finden. Zum anderen können Sie auf die Erfahrung Ihrer Kollegen in verschiedenen Formen, von ganz informeller bis zu einer organisierten Selbsthilfegruppe zurückgreifen. Auch individuelle Supervisionen können hilfreich sein.

Einer der größten Unterschiede zwischen Tier- und Humanmedizin ist, dass die tiermedizinische Behandlung sehr stark von den finanziellen Möglichkeiten des Tierhalters abhängig ist, sodass man nicht allen Patienten die gleiche Therapie anbieten kann. Nicht selten führt es dazu, dass Sie die Behandlung, die am ehesten indiziert wäre, nicht durchführen können oder sogar die laufende Therapie unterbrechen müssen, weil der Tierhalter es nicht mehr bezahlen kann.

Aber auch wenn die finanziellen Mittel da sind, muss man als Tierarzt schwierige Entscheidungen treffen - sei es eine risikoreiche, invasive Behandlung oder das Beenden der Therapie bei palliativen oder schwerstkranken Patienten. Sogar wenn man im Rahmen des sog. „SharedDecision-Making“ (der gemeinsamen Entscheidung von Arzt und Patient bzw. den Angehörigen über die Behandlung) den Tierhalter in den Entscheidungsprozess integriert, trägt man selbst die medizinische Verantwortung. Der Extremfall in der Tiermedizin ist die Euthanasie, die zwar zum Berufsalltag gehört, bei den wenigsten aber zur Routine wird. Um mit solchen schwerwiegenden Entscheidungen gut umgehen zu können, ist es sehr wichtig, dass man sicher ist, dass die getroffene Entscheidung die Richtige ist. Während dies bei Tierärzten mit längerer Berufserfahrung meist der Fall ist, braucht man als Berufsanfänger oft einen Rat - was ein ganz normaler Teil der Weiterbildung und beruflichen Entwicklung ist und wofür man sich nicht schämen muss. Tauschen Sie sich mit Kollegen aus, fragen Sie Ihren (ggf. auch ehemaligen) Chef oder einen Dozenten - niemand nimmt es Ihnen übel und Sie können dadurch Ihre Entscheidung validieren.

\section{Interpersonelle Konflikte}

In der Praxis arbeitet man im Team und es ist nur in den seltensten Fällen möglich, das Praxisleben völlig ohne Konflikte zu führen. Diese können entweder positive Veränderungen als Lösungsansätze mit sich bringen, andererseits aber auch das Arbeitsklima negativ beeinflussen. Deswegen bietet es sich an, Konflikte schnellstmöglich zu erkennen, bevor sie eskalieren. Dazu eignen sich regelmäßige Teambesprechungen, in denen problematische Situationen von allen Beteiligten angesprochen und Lösungsstrategien besprochen werden können. Bei anhaltenden Konflikten kann eine Teamsupervision mit einem externen Supervisor hilfreich sein. Probleme, die einzelne Personen betreffen, sollten mit dem Betroffenen persönlich besprochen werden, ggf. unter Anwesenheit einer 3. Person.

Vermutlich viel häufiger als mit Konflikten im Team werden Sie mit unzufriedenen, fordernden oder unhöflichen Tierhaltern konfrontiert. Während ein gewisses Maß an Unfreundlichkeit akzeptiert werden kann schließlich geht es hauptsächlich um die Behandlung des Tieres und die Klienten sind während des Termins oft sehr aufgeregt - gibt es Situationen, die unzulässig sind und Ihrerseits nicht toleriert werden müssen.

\footnotetext{
Merke

Wenn der Tierhalter verbal aggressiv oder laut wird, sollte das Gespräch lieber beendet werden, um eine weitere Eskalation zu vermeiden.
}

Wenn Sie wissen, dass das Tier länger bei Ihnen in Behandlung bleibt, ist es ratsam, die Situation bei dem darauffolgenden Termin anzusprechen. Sagen Sie dem Tierhalter offen, wenn Sie sein Verhalten nicht nachvollziehen können oder wenn er Sie mit seinen Aussagen verletzt 


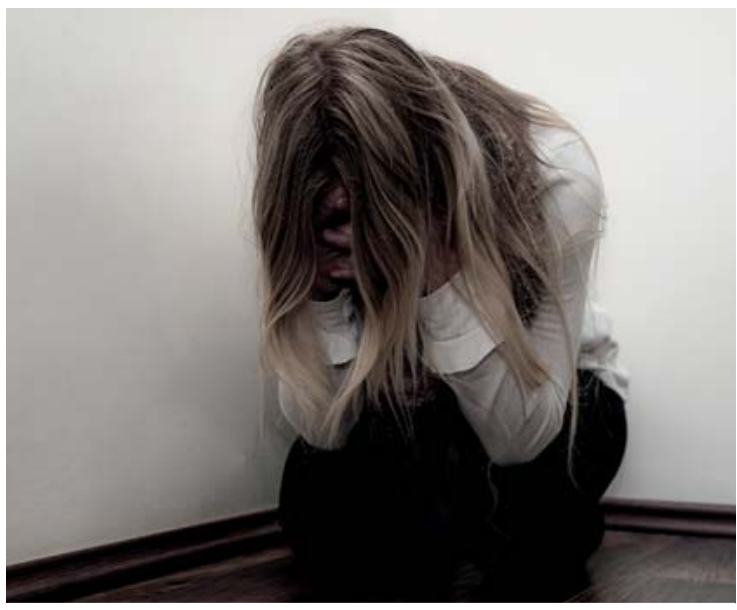

Abb. 2 Wenn Sie länger als 2 Wochen traurig sind oder an Schlafstörungen leiden, dann sollten Sie sich Hilfe holen! Quelle: Kirsten Oborny, Thieme Gruppe

hat. Erklären Sie ihm dabei, dass ein respektvoller Umgang miteinander und eine adäquate Kommunikation wichtig für den Behandlungsprozess des Tieres sind.

\section{Hilfe, Notfall!}

Im tiermedizinischen Bereich wird man immer wieder mit extrem belastenden Situationen konfrontiert. Dies können schwerstverletzte Patienten, Angriffe durch ein Tier oder aggressives Verhalten der Tierhalter sein. Sollte es in Ihrer Praxis zu so einer Situation kommen, ist es wichtig, mit allen Beteiligten zu reden, um zu erfahren, ob jemand Hilfe benötigt. Vergessen Sie dabei sich selbst nicht, insbesondere, wenn es Ihre Praxis ist sind und Sie sich gleichzeitig um Ihr Team kümmern müssen.

Eine nachfolgende Teambesprechung, in der man das Krisenmanagement gemeinsam bespricht, um in der Zukunft in ähnlichen Situationen noch besser reagieren und handeln zu können, erscheint empfehlenswert.

\section{„Brauche ich professionelle Hilfe?"}

Wenn man nach einer besonders stressigen Phase oder einem belastenden Ereignis bei der Arbeit, insbesondere wenn es gleichzeitig auch im privaten Lebensbereich Probleme gibt, einige Tage schlechter schlafen kann, nicht gut gelaunt ist und am liebsten keinen Menschen sehen möchte, ist das meistens eine normale Reaktion, eine Art „seelischer Muskelkater“. Es bietet sich in dieser Zeit trotzdem häufig an, Abstand zu nehmen (beispielsweise durch Mehrarbeitfrei), damit sich der Körper und die Psyche erholen können.

\section{Sollten Sie jedoch mehr als 2 Wochen}

- traurig oder deprimiert sein

- unter Schlafstörungen leiden
- keinen Appetit haben

- sich nicht mehr über positive Erlebnisse freuen können

- sich lust- und energielos fühlen

- sich schlecht konzentrieren können,

suchen Sie medizinische Hilfe auf.

Es ist sehr wichtig, spätestens zu diesem Zeitpunkt „die Notbremse zu ziehen“, denn wenn die psychische Belastbarkeit ohnehin eingeschränkt ist, kann jede zusätzliche Belastung zur Verschlechterung dieser Beschwerden und im schlimmsten Fall zur Entwicklung eines behandlungspflichtigen depressiven Syndroms führen. Auch wenn Sie nicht gern zum Arzt gehen und nicht in der Praxis fehlen wollen, denken Sie daran, dass insbesondere bei anhaltenden Schlafstörungen und verminderter Konzentrationsfähigkeit (diese Symptome treten sehr häufig gemeinsam auf) vermehrt Flüchtigkeitsfehler passieren können, die in der Praxis ungünstige Folgen haben können.

In seltenen Fällen kann es nach besonders belastenden Ereignissen zu einer sog. Traumafolgestörung kommen. Wenn noch mehrere Wochen nach einer Extremsituation Albträume oder plötzlich auftretende bildhafte Erinnerungen (Flashbacks) auftreten und Sie das Gefühl haben, ständig „wie unter Strom zu stehen“, ist es ebenfalls ratsam, einen Arzt aufzusuchen.

Eine weitere Gefahr stellt der Konsum von Alkohol, Medikamenten oder illegalen Substanzen dar, der nicht selten unter den chronisch belasteten Medizinern als „Selbstmedikation“ fungiert. Während ein Glas Wein zur Entspannung am Freitagabend oder eine Schlaftablette pro Monat nicht besorgniserregend sind, sollte man u.a. bei folgenden Anzeichen wachsam werden:

- wenn man die Kontrolle über die konsumierte Menge verliert

- wenn man eine zunehmend höhere Dosis der Substanz benötigt (Dosissteigerung)

- wenn man darüber nachdenkt (oder darauf angesprochen wird) den Konsum einzuschränken oder zu beenden bzw. wenn man es bereits erfolglos versucht hat

" wenn der Konsum verheimlicht wird

\section{WARNANZEICHEN}

Bei Alkohol, Medikamenten oder illegalen Substanzen

- Kontrollverlust über die konsumierte Menge

- Dosissteigerung: eine zunehmend höhere Dosis wird benötigt

- beim Wunsch den Konsum einzuschränken oder zu beenden bzw. wenn man es bereits erfolglos versucht hat

- heimlicher Konsum 
Wenn Sie den Eindruck haben, einen riskanten Substanzkonsum zu betreiben, wenden Sie sich an Ihren Hausarzt oder an eine Suchtberatungsstelle in Ihrer Nähe.

\section{"Ich kann nicht mehr!"}

Einige von Ihnen haben vermutlich schon mal darüber nachgedacht, „das Handtuch zu werfen“ - sei es ein Praxiswechsel oder sogar die Überlegung, „etwas ganz Anderes zu machen “. Im Rahmen der schweren psychischen Belastung kann es im Extremfall aber auch dazu kommen, dass man lebensmüde Gedanken entwickelt. Auch das anhaltende Gefühl der Hoffnungslosigkeit oder eine tiefe Verzweiflung sollten die Alarmglocken läuten lassen.

In diesem Fall ist es wichtig, unverzüglich Hilfe zu suchen. Manchmal hält einen davon der Gedanke ab, dass man nicht der Einzige sei, der solche Gedanken habe oder dass es nur „Spinnerei“ sei. Lassen Sie sich von den Befürchtungen, nicht ernst genommen zu werden, nicht einschüchtern - und nehmen Sie sich selbst ernst.

Dem einen hilft es, sich einer nahestehenden Person anzuvertrauen, der andere redet lieber mit einem Arzt darüber. Heutzutage gibt es auch niederschwellige Hilfsangebote in telefonischer oder elektronischer Form, wo man sich anonym beraten lassen kann. Wichtig ist, die Suizidgedanken direkt anzusprechen. Oft führt allein das Gespräch zu einer deutlichen Entlastung des Betroffenen, ohne dass eine tiefergreifende Behandlung (insbesondere die oft befürchtete Einweisung in eine psychiatrische Klinik) erfolgen muss.

\section{WICHTIC}

Beim Auftreten von Suizidgedanken oder ähnlichen psychischen Beschwerden können Sie sich jederzeit an die Telefonseelsorge unter der bundeseinheitlichen kostenlosen Rufnummer 08 00-1 110111 oder 0800-1110222 und im Internet unter www.telefonseelsorge.de wenden!

\section{Fazit}

Obwohl die Gefahr sehr hoch ist, als Tierarzt belastende Situationen im Arbeitsalltag zu erleben, kann man trotzdem ein ausgeglichenes und erfülltes Berufsleben führen. Hören Sie immer mal auf Ihren Körper, achten Sie auf die Work-Life-Balance und die Psychohygiene und holen Sie sich die entsprechende Unterstützung jedes Mal, wenn Sie es brauchen - mit guten Ressourcen kann man Vieles überstehen!

\section{Korrespondenzadresse}

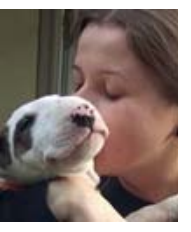

\section{Karolina Kantor}

Fachärztin für Psychiatrie und Psychotherapie Universitätsklinikum Halle (Saale)

Universitätsklinik und Poliklinik für Psychiatrie, Psychotherapie und Psychosomatik Julius-Kühn-Str. 7 06112 Halle/Saale Deutschland

karolina.kantor@uk-halle.de

Literatur

[1] Nett RJ, Witte TK, Holzbauer SM et al. Risk factors for suicide, attitudes toward mental illness, and practice-related stressors among US veterinarians. J Am Vet Med Assoc 2015; 247: 945955

\section{Bibliografie}

Kleintier konkret 2020; 23: 46-49

DOI 10.1055/a-1225-9505

ISSN 1434-9132

(c) 2020. Thieme. All rights reserved.

Georg Thieme Verlag KG, Rüdigerstraße 14,

70469 Stuttgart, Germany 